\title{
Fomentar la salud mediante las alianzas de investigación en América Latina*
}

\author{
Jaime Miranda1,2, Ana Cristina Castro-Ávila ${ }^{3,4}$ y Luis Alejandro Salicrup ${ }^{5,6}$
}

\begin{abstract}
Jaime Miranda y cols. sostienen que las alianzas son fundamentales para fortalecer y mantener la capacidad de investigación en materia de salud en América Latina
\end{abstract}

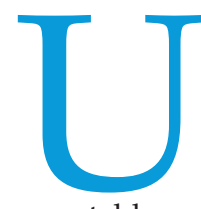
no de los objetivos principales de la Política de investigación para la salud ${ }^{1}$ de la Organización Panamericana de la Salud (OPS) es establecer alianzas con el fin de ejecutar estrategias de salud apropiadas y sostenibles. Esta política procura facilitar el desarrollo y la disponibilidad a mayor escala de productos y tecnologías asequibles y accesibles pertinentes para la respuesta a los retos de salud pública de la Región de las Américas; crear grupos de investigación en los Estados Miembros de la OPS con competencias que permitan el intercambio de los conocimientos procedentes de la investigación; y promover la colaboración entre los diversos interesados directos, incluidos el público general, el gobierno y los sectores académico y privado a fin de hacer frente a las prioridades nacionales y regionales. ${ }^{1}$

Los intentos de crear alianzas en el continente se remontan a los años setenta. Por ejemplo, la UNESCO, el Programa de las Naciones Unidas para el Desarrollo y otros organismos de las Naciones

\footnotetext{
* Traducción oficial al español efectuada por la Organización Panamericana de la Salud. En caso de discrepancia, prevalecerá la versión original en inglés.

1 Profesor de investigación ${ }^{1}$ Facultad de medicina, Universidad Peruana Cayetano Heredia, Avenida Honorio Delgado 430, San Martín de Porres, Lima 31, Perú. $₫$ Jaime.Miranda@upch.pe
}

Unidas financiaron alianzas Sur Sur entre instituciones de América Latina con el objeto de apoyar las redes de investigación en biología, microbiología y ciencias biomédicas. $^{2}$ El objetivo era fomentar el intercambio de conocimientos y crear capacidad de investigación en los países con infraestructuras de investigación poco desarrolladas, al capacitar a sus científicos en instituciones más grandes y con mayor experiencia en países como Argentina, Brasil y México. Sin embargo,

\section{MENSAJES CLAVE}

- Las alianzas han sido y seguirán siendo primordiales para el avance de la investigación en salud de gran calidad en América Latina y el mundo.

- La política de investigación para la salud de la OPS rige en América Latina y presta apoyo decidido a la promoción de las alianzas.

- Las alianzas de investigación en la región deben vincular entes iguales que comparten objetivos y metas definidos de común acuerdo.

- Para que las alianzas sean eficaces, los interesados directos deben ponerse de acuerdo sobre una visión y una misión comunes; negociar las funciones, responsabilidades y acciones; definir los objetivos; y establecer los procedimientos de negociación y de participación en el poder.

- Las alianzas no son todas iguales y deben evolucionar en función de sus puntos fuertes específicos.

- América Latina tendrá que adaptarse a un mundo en evolución mediante el intercambio de la información. Las iniciativas de mancomunación de datos representan un reto para los paradigmas de colaboración existentes, por lo que un marco de referencia para las alianzas puede ayudar a definir el camino que se debe seguir en la región.

\footnotetext{
Profesor de investigación ${ }^{1}$ CRONICAS Centro de excelencia en las Enfermedades Crónicas, Universidad Peruana Cayetano Heredia, Miraflores, Lima 18, Perú.

Investigador. Departamento de Ciencias de la Salud, Universidad de York, York, Yorkshire 5DD YO10, Reino Unido.

4 Investigador. Carrera de Kinesiología, Facultad de Medicina, Universidad del Desarrollo, Clínica Alemana, Santiago 7610658, Chile.
}

\footnotetext{
Asesor principal para la Investigación Mundial en Salud Organización Panamericana de la Salud/ Organización Mundial de la Salud, Washington, D.C. 20037-2895, Estados Unidos.

6 Asesor principal para la Investigación Mundial en Salud Centro para la Salud Mundial, Instituto Nacional del Cáncer, Institutos Nacionales de Salud de los Estados Unidos, Bethesda, Maryland, Estados Unidos.
} 
muchas redes se desintegraron debido a la falta de financiamiento nacional. Algunos decenios después, están surgiendo ejemplos dignos de mención de colaboraciones y alianzas Norte Sur y Sur Sur.

Los términos "alianza" y "colaboración" a menudo se usan indistintamente, pero las relaciones son diferentes. ${ }^{3} \mathrm{La}$ colaboración implica que las partes trabajan juntas en un cometido intelectual, mientras que la alianza significa una relación entre iguales que tienen objetivos y metas definidos de común acuerdo. ${ }^{3}$

La creación, la puesta en marcha y el mantenimiento de las alianzas pueden plantear dificultades y es necesario comprender los factores que las obstaculizan o las facilitan. Los obstáculos culturales y la compartimentación de los conocimientos también pueden entorpecer la construcción de alianzas útiles. En el presente artículo se exploran algunas características de las alianzas de investigación existentes, que aprovechan al máximo las oportunidades de financiamiento $y$ están logrando compartir los recursos, las metas y los objetivos en América Latina. Se escogieron estudios de casos que destacan las interacciones interdisciplinarias, interinstitucionales, Norte Sur, Sur Sur, y de la comunidad académica y la industria.

\section{ALIANZAS PARA GENERAR NUEVOS CONOCIMIENTOS}

El National Heart, Lung, and Blood Institute (NHLBI) y el United Health Group de los Estados Unidos han señalado la necesidad apremiante de crear capacidad e infraestructura para la investigación, con el fin de responder al gran reto que representa la carga de enfermedad cada vez mayor por las enfermedades no transmisibles en los países de ingresos bajos y medianos. ${ }^{4}$ Juntos, pusieron en marcha la iniciativa por las enfermedades crónicas, que funcionó entre el 2009 y el 2014 con el fin de prestar apoyo a una red de once centros de investigación en diez países de ingresos bajos y medianos, cuatro de ellos en América Latina (Argentina, Guatemala, México y Perú). ${ }^{4}$ Cada centro de excelencia asoció al menos una institución académica de ingresos altos en Australia, Canadá, Estados Unidos o Europa con una organización local, que podía ser una universidad o una institución dedicada a la investigación o la atención de salud. ${ }^{4}$ Los países de ingresos bajos y medianos fueron los principales destinatarios de las subvenciones concedidas por el programa, lo cual significó que muchos países tuvieron que alcanzar un rápido desarrollo científico y lograr que sus procedimientos administrativos y de gestión de las subvenciones cumpliesen con las normas internacionales. ${ }^{4}$

Al 2016, los cuatro centros de excelencia en América Latina habían participado en 14 proyectos colaborativos con diversos diseños de investigación y habían producido 86 publicaciones y 97 presentaciones de investigación, y capacitado a 216 investigadores de posgrado. ${ }^{4}$ Además, esta alianza facilitó un proceso de armonización de datos que hizo posible la exploración de nuevos temas de investigación centrados en las enfermedades no transmisibles en los centros de excelencia de la región.

Los centros de excelencia en América Latina y sus asociados mundiales se beneficiaron mutuamente con el programa, no solo por los progresos científicos sino también por la interacción a nivel personal e institucional a todo lo largo del proceso desde la concepción de la investigación hasta la difusión de sus resultados. Los centros de excelencia para las enfermedades crónicas no transmisibles lograron su objetivo al fortalecer la capacidad de investigación, compartir los datos y las redes, promover la mancomunación de los beneficios y los méritos, y transformar un esfuerzo único de financiamiento en un emprendimiento a largo plazo. Lamentablemente, la falta de financiamiento sostenido puede afectar las interacciones en curso y poner en riesgo la alianza y sus cometidos de investigación. Esta situación destaca la importancia de prestar apoyo a las redes que trabajan en consonancia con las necesidades actuales de investigación y que estudian la salud desde una perspectiva multidisciplinaria y de multimorbilidad, en lugar de trabajar en forma aislada. ${ }^{5}$

\section{ALIANZAS QUE PROMUEVEN LA PARTICIPACIÓN DE LOS INTERESADOS DIRECTOS}

Los investigadores de la región rara vez cuentan con la oportunidad y el apoyo de los gobiernos para probar y recomendar intervenciones de salud pública. El proyecto GUIA (por su sigla en inglés) de intervenciones útiles para la actividad física puesto en marcha en Brasil y América Latina es una alianza de investigación multidisciplinaria transnacional que ha adoptado un enfoque diferente.
En lugar de evaluar nuevas intervenciones por medio de ensayos aleatorizados y luego ampliar a mayor escala las iniciativas que son eficaces, el proyecto evalúa las intervenciones de salud pública que ya se ejecutan en América Latina.

Mediante un mecanismo iterativo de evaluación, cotejo de los datos de las investigaciones y adaptación de las intervenciones, el proyecto GUIA ha realizado evaluaciones por medio de la investigación académica de diferentes programas de salud pública, que llevaron a que Brasil ampliase la escala de aplicación federal y nacional de programas que ofrecen clases de actividad física en la comunidad (Academia da Cidade) y de programas que estimulan la actividad física con provisión de espacios y recursos públicos (Academia da Saúde). Asimismo, se está evaluando y ampliando la escala de programas equivalentes en otros países como Colombia y Chile. ${ }^{6}$

El proyecto GUIA fue financiado y coordinado por los Centros para el Control de Enfermedades y el Centro de Investigación en Prevención en Saint Louis (Estados Unidos), conjuntamente con tres universidades de Brasil. ${ }^{6}$ El proyecto utilizó los vínculos de salud pública y las colaboraciones establecidas entre los gobiernos de ambos países que ya existían antes de iniciar el proyecto. Se invitó a participar en el proyecto a una red diversa de asociados en la comunidad académica, las instituciones gubernamentales (de todos los niveles) y no gubernamentales (por ejemplo, la OPS y el Centro de Estudos do Laboratório de Aptidão Física de São Caetano do Sul o CELAFISC). Un factor importante en su éxito fue la forma como el proyecto abordó la sensibilidad cultural, lo que permitió a los asociados reconocer y dar prioridad a las iniciativas de investigación en función del contexto local. ${ }^{6}$

El proyecto GUIA ejemplifica la importancia de interactuar con los interesados directos desde el principio, fortaleciendo la capacidad de los investigadores y los responsables de formular políticas para utilizar y generar evidencia basada en la práctica. Se ha reconocido que un aspecto importante de la alianza ha sido la difusión de los resultados para que los utilicen las entidades regionales y los apliquen en los programas. ${ }^{6}$

Algunas reflexiones sobre el éxito del proyecto GUIA también han resaltado la importancia de escoger el momento oportuno. El proyecto empezó cuando los 
organismos brasileños de salud pública estaban preparados para abordar iniciativas de prevención de las enfermedades no transmisibles ${ }^{6}$ y en un momento en el cual surgía un grupo de investigadores jóvenes con talento en América Latina, ${ }^{7}$ con lo cual se demostró que el hecho de invertir en los investigadores desde el principio de su carrera puede dar lugar a iniciativas de mayor magnitud. Sin embargo, retener a los investigadores con talento exige una inversión mayor. Sin este apoyo, será difícil mantener un caudal de talento para trabajar, innovar e impulsar las iniciativas de salud pública a fin de abordar las enfermedades no transmisibles en la región. Es primordial contar con una masa crítica de talento, a fin de utilizar su experiencia para promover las enseñanzas o sostener el compromiso con los conocimientos y las políticas en los diversos niveles.

\section{ALIANZAS PARA LOGRAR TECNOLOGÍAS MÁS APROPIADAS Y ASEQUIBLES}

Al transferir las innovaciones de una región a otra no siempre se obtiene el resultado previsto, y el desarrollo de nuevas tecnologías médicas requiere tiempo y dinero. Por consiguiente, las alianzas serán esenciales para que se apliquen tecnologías médicas apropiadas y asequibles en América Latina. Las alianzas entre la comunidad académica y la industria, incluidas las colaboraciones Norte Sur y Sur Sur, podrían contribuir al logro de intervenciones que sean más costo-eficaces, apropiadas, asequibles y sostenibles frente a los principales retos de salud pública del continente. Además, al conseguir la participación de múltiples entidades dentro de cada institución y entre diversas instituciones, estas alianzas pueden ayudar a crear capacidad de investigación interdisciplinaria y a formar científicos en América Latina que se dediquen a la investigación, el desarrollo y la innovación.

Un ejemplo en la región de estímulo para crear alianzas en favor de la investigación para la innovación en la salud mundial es el financiamiento basado en la convocatoria de concursos públicos de los Institutos Nacionales de Salud (INS) de los Estados Unidos por intermedio de su Instituto Nacional del Cáncer (INC). Estas alianzas son especialmente pertinentes dada la carga de enfermedad en la región: 85\% de los casos de cáncer cervicouterino en el mundo ocurren en los países de ingresos bajos y medianos y $16 \%$ de ellos en el continente americano. ${ }^{8}$ Una de las alianzas respaldada en la actualidad por el financiamiento de los Institutos Nacionales de Salud/ Instituto Nacional del Cáncer incluye instituciones públicas y privadas en América Latina y los Estados Unidos que abordan el uso accesible y asequible de medios de diagnóstico moleculares en la detección temprana de los subtipos del virus del papiloma humano (VPH) que pueden causar cáncer cervicouterino. En concreto, los investigadores de América Latina y el Centro Internacional de Investigaciones sobre el Cáncer trabajan con el fin de desarrollar una prueba de VPH basada en la detección de la actividad de la oncoproteína E6/E7, que es una proteína presente en los ocho subtipos de $\mathrm{VPH}$ asociados con mayor frecuencia con el cáncer cervicouterino. En el estudio se utilizarán redes de investigación clínica preexistentes en América Latina con el fin de evaluar la utilidad de la prueba como herramienta ordinaria de detección sistemática del cáncer cervicouterino. ${ }^{9}$ Otro ejemplo de una alianza apoyada por este programa que aprovecha las redes existentes en América Latina trabaja para transformar un dispositivo de ablación que no utiliza gases, el sistema crioquirúrgico CryoPen(R), en un dispositivo de crioterapia portátil y de bajo costo que pueda ser utilizado por cualquier prestador de atención de salud. ${ }^{10}$

Las alianzas entre la industria, la comunidad académica y el gobierno, según se describen en la política de investigación para la salud, se basan en la elaboración de una agenda conjunta que aclare las responsabilidades, fortalezca la capacidad y el uso compartido de los datos y las redes a fin de lograr un impacto duradero en los retos de salud pública actuales y emergentes. Sin embargo, estas alianzas podrían estar en riesgo si los diversos organismos no pueden articular o armonizar sus prioridades y si los cambios en las agendas políticas pueden crear obstáculos. La creación de estas alianzas depende de las oportunidades de financiamiento existentes y futuras. A menos que los asociados participantes cuenten con un plan sostenido de desarrollo de productos, existe el riesgo de que cuando termine el financiamiento cesen las interacciones facilitadas por las alianzas, con lo cual se pone en riesgo el proceso de investigación e innovación y, por consiguiente, sus productos previstos.

\section{DISCUSIÓN}

Las alianzas multidisciplinarias promueven una mejor comprensión entre los interesados directos de los sectores público y privado en el plano nacional e internacional. Estas alianzas son fundamentales para hacer frente a los retos de salud actuales y futuros: el tratamiento de las enfermedades transmisibles y las no transmisibles, el mantenimiento de las intervenciones de salud pública y la creación de soluciones innovadoras a los problemas comunes que se pueden aplicar en los países de ingresos bajos y medianos.

Para que las alianzas sean eficaces, los interesados directos deben estar de acuerdo en una visión y una misión comunes; negociar las funciones, responsabilidades y acciones; definir los objetivos; y establecer los procedimientos de negociación y de participación en el poder. ${ }^{11}$ Es necesario crear alianzas estratégicas con todos los posibles interesados directos (gobiernos, universidades, sector privado, financiadores, organizaciones internacionales, asociaciones profesionales, organizaciones comunitarias y grupos de pacientes) con el fin de abordar las prioridades nacionales mediante la investigación para la salud. Las alianzas entre diferentes interesados directos son también importantes para el logro de los objetivos de la política de investigación para la salud, es decir, mejorar la equidad, la salud y el desarrollo. ${ }^{12}$

Las alianzas de investigación incipientes a menudo fracasan debido a presupuestos poco realistas y a la falta de liderazgo, negociación, trabajo en equipo y aptitudes de gestión. En la actualidad, los investigadores en América Latina adquieren estas aptitudes "con la práctica", de manera tardía en su carrera profesional. La introducción y el fortalecimiento de estas competencias durante la capacitación de grado y posgrado podrían facilitar el establecimiento de relaciones duraderas y sostenibles entre pares, lo que es un requisito para la creación de alianzas productivas. La incorporación de estas aptitudes en el programa de estudios requerirá otra alianza: entre las instituciones académicas y los gobiernos en favor del fortalecimiento de las capacidades.

En América Latina se puede aprovechar la diversidad de su población y los idiomas comunes, especialmente el español y el portugués, con el fin de construir alianzas que difícilmente funcionarían de otra manera. Es necesario adaptarse 
pues los desafíos existentes (o sea, la agenda inconclusa de las enfermedades infecciosas, el fortalecimiento de los sistemas de salud y el logro de la atención de salud para todos) se aunarán a nuevos retos diferentes como la multimorbilidad, las enfermedades no transmisibles y los enfoques del uso de grandes volúmenes de datos (big data) en la investigación, temas todos que se podrán abordar mejor por medio de alianzas exitosas. ${ }^{13}$ La política de investigación para la salud de la OPS ofrece una base para incluir y alinear múltiples organismos dedicados a una meta común: la investigación para la salud y la equidad. Estas alianzas y sus resultados serán cruciales en el fortalecimiento de los sistemas de salud que abordan los mayores retos de salud pública. También facilitarán la evaluación comparativa de los adelantos en materia de salud, de manera que los países puedan hacer planes fundamentados en la evidencia con el fin de mejorar la calidad general de los servicios de salud y el acceso a ellos, dos de los pilares principales de la estrategia de salud universal con cuya ejecución se han comprometido la OPS y todos los Estados Miembros.

Agradecimientos. A Luis Gabriel Cuervo por sus ideas y sugerencias para este artículo y a Miguel Ossandon por el valioso intercambio de información acerca del alcance del programa del INC sobre tecnologías asequibles del cáncer para los países de ingresos bajos y medianos. A Samantha Brew por su ayuda con la edición de las versiones iniciales del manuscrito.

Conflicto de intereses. Después de haber leído y comprendido la política de The BMJ sobre conflicto de intereses, los autores declaran los siguientes intereses: JJM ha sido miembro del Comité Asesor de Investigaciones en Salud de la OPS (CAIS) desde septiembre del 2014 y presidió dicho comité desde septiembre del 2014 hasta abril del 2016, además fue investigador principal en el Centro de Excelencia Peruano financiado por el NHLBI. LAS ha trabajado en el Instituto Nacional del Cáncer/Institutos Nacionales de Salud y la OPS/OMS.

Contribución de los autores. JJM concibió la idea y la estructura del artículo y redactó la primera versión con la asistencia de ACCA y LAS. ACCA elaboró la primera revisión bibliográfica, contribuyó a la redacción del manuscrito y formuló observaciones sobre todas las versiones y revisiones. Todos los autores contribuyeron con aportaciones intelectuales, editaron el manuscrito y aprobaron su versión definitiva.

Procedencia del artículo y revisión por pares. Comisionado; sin revisión por pares externa.

Declaración. Las opiniones expresadas en este manuscrito son responsabilidad del autor y no reflejan necesariamente los criterios ni la política de la RPSP / PAJPH y/o de la OPS/OMS.

Serie Fortalecimiento de la investigación para la salud en las Américas. Este artículo es parte de una serie propuesta por la Organización Panamericana de la Salud (OPS) y comisionada por The $B M J$, que tuvo a cargo la revisión por pares y la edición, y tomó la decisión sobre la publicación de los artículos sin participación de la OPS. Los gastos de publicación en acceso abierto de la serie original en inglés fueron financiados por la OPS. Acceso a la serie original en inglés en www .bmj.com/health-research-americas; acceso a la serie en español y portugués en https://www.paho.org/journal/es /numeros-especiales / fortalecimiento -investigacion-para-salud-americas.

\section{REFERENCIAS}

1. Organización Panamericana de la Salud. Política de investigación para la salud (documento CD49/10). OPS/OMS, 2009. https://www.paho.org/hq/images/stories /KBR/Research/politica\%20de\%20investigacion\%20para\%20la\%20salud.pdf.

2. Sobre RELAB. Red Latinoamericana de Ciencias Biológicas. http://relab.biologia .ucr.ac.cr/

3. Glazer G, Alexandre C, Reid Ponte P. Legislative: Partnership or collaboration: words matter. Online Journal of Issues in Nursing 2008;13. https://www.nursingworld.org /MainMenuCategories/ANAMarketplace /ANAPeriodicals/OJIN/TableofContents /vol132008/No2May08/Partnershipor Collaboration.html

4. Engelgau MM, Sampson UK, Rabadan-Diehl C, et al, National Heart, Lung, and Blood Institute-United Health Global Health Centers of Excellence Collaborators. Tackling NCD in LMIC: achievements and lessons learned from the NHLBIUnitedHealth Global Health Centers of Excellence Program. lob Heart 2016;11:515. doi:10.1016/j. gheart.2015.12.016

5. Academy of Medical Sciences. Addressing the global challenge of multimorbidity: Lessons From BRICS countries. Academy of
Medical Sciences, 2017. https://acmedsci .ac.uk/ file-download/3855485

6. Parra DC, Hoehner CM, Hallal PC, et al. Scaling up of physical activity interventions in Brazil: how partnerships and research evidence contributed to policy action. Glob Health Promot 2013;20:5-12. doi:10.1177/1757975913502368

7. Pratt M, Brownson RC, Ramos LR, et al. Project GUIA: A model for understanding and promoting physical activity in Brazil and Latin America. J Phys Act Health 2010;7(Suppl 2):S131-4. doi:10.1123 /jpah.7.s2.s131

8. International Agency for Research on Cancer. GLOBOCAN 2012 cervical cancer: Estimated incidence, mortality and prevalence worldwide in 2012. http:// globocan.iarc.fr/old/FactSheets/cancers /cervix-new.asp

9. Instituto Nacional del Cáncer (Estados Unidos). Supporting the development of affordable technologies for cancer detection, diagnosis, and treatment in LMICs. 2016. https://www.cancer.gov/about-nci /organization/cgh/blog/2016

10. NIH RePORTER. CryoPen: an innovative treatment for cervical precancer in lowresource setting. NIH Research Portfolio
Online Reporting Tools. https://project reporter.nih.gov/project_info_description .cfm?projectnumber $=5$ UH3CA189883-05

11. Gallant MH, Beaulieu MC, Carnevale FA. Partnership: an analysis of the concept within the nurse-client relationship. J Adv Nurs 2002;40:149-57. doi:10.1046/j.1365-2648.2002.02357.x

12. Organización Panamericana de la Salud/ Organización Mundial de la Salud. Milestones for a Policy on Research for Health. 2014. https://www.paho.org /hq/dmdocuments/2014/Timeline-Research -Spanish.pdf

13. Sterman JD. Learning from evidence in a complex world. Am J Public Health 2006;96:505-14. doi:10.2105/AJPH.2005 .066043

Forma de citar (artículo original): BMJ 2018;362:k2690 doi: http://dx.doi.org/10.1136/bmj.k2690

Manuscrito original en inglés publicado el 16 de julio de 2018. 